\title{
CYCLIC HILBERT SPACES AND CONNES' EMBEDDING PROBLEM
}

\author{
Valerio CAPRARO and Florin RĂDULESCU
}

May 5, 2018

\begin{abstract}
Let $M$ be a $I I_{1}$-factor with trace $\tau$, the linear subspaces of $L^{2}(M, \tau)$ are not just common Hilbert spaces, but they have additional structure. We introduce the notion of a cyclic linear space by taking those properties as axioms. In Sec.2 we formulate the following problem: "does every cyclic Hilbert space embed into $L^{2}(M, \tau)$, for some $M ? "$. An affirmative answer would imply the existence of an algorithm to check Connes' embedding Conjecture. In Sec.3 we make a first step towards the answer of the previous question.
\end{abstract}

\section{Contents}

1 Cyclic Hilbert spaces

2 Relation with Connes' embedding conjecture

3 Extension of cyclic vector spaces

4 Problems we were not able to solve

\section{Cyclic Hilbert spaces}

Let $M$ be a finite factor with unique normalized trace $\tau$ and let $L^{2}(M, \tau)$ be the Hilbert space obtained by taking the closure of the vector space $M$ with respect to 
the inner product $(x, y)=\tau\left(y^{*} x\right)^{\frac{1}{2}}$. Consider a finite-dimensional real Hilbert subspace $H \subseteq M_{s a} \subseteq L^{2}(M, \tau)$ containing the identity. Observe that $H$ is not just a common Hilbert space, but it has additional structure.

Proposition 1. The mapping $\ll a \otimes b, c \otimes d \gg \doteq \tau(a b d c)$ is a bilinear hermitian positive form on $(H \otimes H) \otimes \mathbb{C}$ and the following properties are satisfied

1. the mappings $v \rightarrow v \otimes 1$ and $v \rightarrow 1 \otimes v$ are isometric embeddings, i.e.

$$
(v, v)=\ll v \otimes 1, v \otimes 1 \gg=\ll 1 \otimes v, 1 \otimes v \gg
$$

2. $\ll \cdot, \cdot \gg$ is cyclic in the following sense

$$
\ll a \otimes b, c \otimes d \gg=\ll c \otimes a, d \otimes b \gg
$$

3. $\ll \cdot, \cdot \gg$ is self-adjoint in the following sense

$$
\ll a \otimes b, c \otimes d \gg=\overline{\ll b \otimes a, d \otimes c \gg}
$$

4. $\ll \cdot, \cdot \gg$ verifies the following property

$$
\ll a \otimes b, c \otimes d \gg=\ll b \otimes d, a \otimes c \gg
$$

5. The mapping $J_{H}:(H \otimes H) \otimes \mathbb{C} \rightarrow(H \otimes H) \otimes \mathbb{C}$ defined by setting $J_{H}(a \otimes b)=b \otimes a$ is an isometric involution, i.e.

$$
\begin{aligned}
& \text { (a) } J_{H}\left(J_{H}(a \otimes b)\right)=a \otimes b \\
& \text { (b) } \ll J_{H}(a \otimes b), J_{H}(a \otimes b) \gg=\ll a \otimes b, a \otimes b \gg
\end{aligned}
$$

In this article we want to consider Hilbert spaces which verify these five additional properties. Before giving the definition let us observe that properties 2. and 3. together imply properties 4 . and 5. Indeed

Lemma 2. Let $(H,(\cdot, \cdot), \ll \cdot, \cdot \gg)$ be a Hilbert space equipped with a bilinear positive hermitian form $\ll \cdot, \cdot \gg$ on $(H \otimes H) \otimes \mathbb{C}$. If $\ll \cdot, \cdot \gg$ verifies 2. and 3. then it verifies also 4. and 5. 
Proof. Suppose 2. and 3. are verified. Applying hermitianity, 2. and hermitianity again we get 4 . Indeed

$$
\begin{gathered}
\ll a \otimes b, c \otimes d \gg=\overline{\ll c \otimes d, a \otimes b \gg}=\overline{\ll a \otimes c, b \otimes d \gg}= \\
=\ll b \otimes d, a \otimes c \gg
\end{gathered}
$$

On the other hand, applying 3. and hermitianity, we get 5. Indeed

$$
\begin{aligned}
\ll J_{H}(a \otimes b), J_{H}(a \otimes b) & =\ll b \otimes a, b \otimes a \gg=\overline{\ll a \otimes b, a \otimes b \gg}= \\
= & \ll a \otimes b, a \otimes b \gg
\end{aligned}
$$

Notice that we have not used the completeness with respect to $(\cdot, \cdot)$. Thus we can give the following

Definition 3. A cyclic pre-Hilbert space is a quadruple $(V,(\cdot, \cdot), 1, \ll \cdot, \cdot \gg)$, where $(V,(\cdot, \cdot))$ is a real pre-Hilbert space, $1 \in V$ is a pointed vector such that $(1,1)=\|1\|^{2}=1$ and $\ll \cdot, \cdot \gg$ is a bilinear complex-valued, hermitian positive form on $(V \otimes V) \otimes \mathbb{C}$ verifying properties 1.,2. and 3. (and, consequently, 4. and 5.).

\section{Relation with Connes' embedding conjecture}

We have begun studying cyclic spaces motivated by Connes' embedding conjecture. Before explaining how they are related to each other, let us briefly recall Connes' embedding conjecture. Let $R$ be the hyperfinite $I I_{1}$ factor (with unique trace denoted by $\tau$ ) and let $\omega \in \beta(\mathbb{N}) \backslash \mathbb{N}$ be a free ultrafilter on the natural number. One can construct the ultrapower $R^{\omega}$ in the following way: first consider $l^{\infty}(R)=\left\{\left(x_{n}\right)_{n} \subseteq R: \sup _{n}\left\|x_{n}\right\|<\infty\right\}$; then consider its ideal $I_{\omega}=\left\{\left(x_{n}\right)_{n} \in l^{\infty}\left(R^{\omega}\right): \lim _{n \rightarrow \omega} \tau\left(x_{n}^{*} x_{n}\right)^{\frac{1}{2}}=0\right\}$; finally consider the quotient $R^{\omega}=l^{\infty}(R) / I_{\omega}$. It turns out to be a non weakly separable $I I_{1}$ factor with trace $\tau_{R^{\omega}}\left(x+I_{\omega}\right)=\lim _{n \rightarrow \omega} \tau\left(x_{n}\right)$, where $\left(x_{n}\right)$ is any representative sequence for $x$. Connes' embedding conjecture states that any $I I_{1}$-factor with separable predual embeds into $R^{\omega}$ $([\mathrm{Co}]$. This conjecture has become more and more interesting in recent years, since many authors have found lots of equivalent conditions showing that this conjecture is linked to 
several branches of mathematics (like group theory and metric geometry), besides being transversal to most of the sub-specializations of Operator Algebras (see [Br], [Br2], Ca-Pa], Co-Dy], [El-Sz], [Ha-Wi2], [Ki], [Ne-Th], Oz], Pe], Ra1], Ra2], Ra3], Vo2], Vo3] for some reference). Here is the problem we want to focus

Problem 4. Does every separable cyclic space embed into some $I I_{1}$-factor with separable predual?

We are interested in this problem because an affirmative answer would imply the existence of an algorithm to check Connes' embedding conjecture. Indeed

1. Take a $I I_{1}$-factor with separable predual $M$. If Prob 4 has affirmative answer, then we could theoretically enumerate all the inequalities verified by the moments of order 3 and 4 in $M$. They are positive definite polynomials of degree less than or equal to 4 , that are quite easy to understand, being exactly the inequalities of a cyclic space.

2. Take these polynomials and calculate their own infimum on positive matrices of order $n$. Let $\varepsilon_{n} \geq 0$ be such an infimum. Observe that Connes' embedding conjecture is true if and only if $\varepsilon_{n}$ converges to 0 , when $n$ goes to infinity. Indeed Connes' embedding problem has an affirmative answer if and only if one can approximate the moments of order 3 and 4 (see [Ra3]).

If the Connes embedding conjecture is true then the algorithm is infinite for at least one $M$. On the other hand, if it finite for some $M$, that is, it stops after a finite time, then the Connes embedding conjecture might be true or false. In this case, the algorithm could be used as a tool for constructing possible counter-example.

\section{Extension of cyclic vector spaces}

The idea to answer Prob 4 is the following: suppose we have an orthonormal basis $\left\{x_{n}\right\}$ for $L^{2}(M, \tau)$, then we would have

$$
x_{i} x_{j}=\sum_{n} \alpha_{i j}^{n} x_{n}
$$

and thus the first requirement is that an element of $V \otimes V$ should be actually an element of $V \otimes 1$. It means that the first step is to extend the cyclic structure by adjoining elements. 
More precisely we have to extend the cyclic structure on $V$ to a cyclic structure on a space $W$ of the shape $V \oplus \mathbb{R} Y$, where $Y$ is an indeterminate, in order to reconstruct step by step the product. We mean that, chosen arbitrarily $y \in(V \otimes V) \otimes \mathbb{C}, y$ has to be represented as the indeterminate $Y$, i.e. $y=1 \otimes Y=Y \otimes 1$. This is why extending the cyclic scalar product $\ll \cdot, \cdot \gg$ to one over $((V \oplus \mathbb{R} Y) \otimes(V \oplus \mathbb{R} Y)) \otimes \mathbb{C}$ (where $Y$ represents an arbitrary element in $V \otimes V$ ) means exactly that we are extending the scalar product on $V$ to get the fixed product $y$ of elements in $V$. Such a purpose forces some necessary assumptions on $y$ :

1. $y$ must be self-adjoint, in the sense that $J_{V} y=y$.

2. $y$ must have norm 1, i.e. $\ll y, y \gg=1$.

3. $y$ is not an element of $V \otimes 1$ or $1 \otimes V$ (otherwise we would have trivial product). In this case we say that $y$ is a non-trivial element in $V \otimes V$.

Unfortunately we are not able to extend the structure exactly, but just approximately.

Definition 5. Let $V$ be a finite dimensional cyclic vector space with orthonormal basis $\left\{x_{1}, \ldots x_{n}\right\}$. An $\varepsilon$-perturbation of the original scalar product $\ll \cdot, \cdot \gg$ is another scalar product $\ll \cdot, \cdot \gg_{\varepsilon}$ such that

$$
\left|\ll x_{i} \otimes x_{j}, x_{k} \otimes x_{l} \gg-\ll x_{i} \otimes x_{j}, x_{k} \otimes x_{l} \gg_{\varepsilon}\right|<\varepsilon \quad \forall i, j, k, l \in\{1, \ldots n\}
$$

Proposition 6. Let $V$ be a finite dimensional cyclic vector space and $y$ a self-adjoint and non-trivial element in $(V \otimes V) \otimes \mathbb{C}$ with norm 1. For every $\varepsilon>0$, there exists an $\varepsilon$-perturbation of $\ll \cdot, \cdot \gg$ which extends to a cyclic structure on $W:=V \oplus \mathbb{R} Y$ with the property $y=1 \otimes Y=Y \otimes 1$.

Proof of this proposition is quite technical, so we will divide it in several steps. Indeed, let $1, x_{2} \ldots x_{n}$ an orthonormal basis of $V$ ( 1 is the pointed vector on $V$ ), we need to define the products

$$
\begin{array}{lll}
\ll Y \otimes x_{i}, x_{j} \otimes x_{l} \gg_{\varepsilon} & \ll Y \otimes x_{i}, Y \otimes x_{j} \gg_{\varepsilon} & \ll Y \otimes Y, x_{i} \otimes x_{j} \gg_{\varepsilon} \\
\ll Y \otimes x_{i}, x_{j} \otimes Y \gg_{\varepsilon} & \ll Y \otimes x_{i}, Y \otimes Y \gg_{\varepsilon} & \ll Y \otimes Y, Y \otimes Y \gg_{\varepsilon}
\end{array}
$$


The remaining products $\ll x_{i} \otimes x_{j}, x_{k} \otimes x_{l} \gg_{\varepsilon}$ will be defined in the course of the proof, when we find the suitable $\varepsilon$-perturbation of $\ll \cdot, \cdot \gg$. The most technical part of the proof is the definition of $\ll Y \otimes x_{i}, x_{j} \otimes x_{k} \gg_{\varepsilon}$, which will be the first and second step. In the first step we follow a sequence of necessary conditions in order to construct a linear system whose solutions allow us to define such products; in the second step we solve this linear system. Before going into the first step, let us state some preliminary notions.

By the fifth property in Prop $1, J_{V}$ behaves on $(V \otimes V) \otimes \mathbb{C}$ like an involution, so it is natural to fix the following terminology.

Definition 7. An element $x \in(V \otimes V) \otimes \mathbb{C}$ is called self-adjoint if $J_{V} x=x$. For an element $x \in(V \otimes V) \otimes \mathbb{C}$ which is not self-adjoint, its real part is $\operatorname{Re}(x)=\frac{x+J_{V} x}{2}$ and the imaginary part is $\operatorname{Im}(x)=\frac{x-J_{V} x}{2 i}$.

\section{Step 1}

Let $P$ be the projection of $(V \otimes V) \otimes \mathbb{C}$ onto the first $(V \otimes \mathbb{C})^{\perp}$. Observe that

$$
\begin{gathered}
\ll Y \otimes x_{i}, x_{j} \otimes x_{k} \gg_{\varepsilon}=\ll Y \otimes x_{i}, P\left(x_{j} \otimes x_{k}\right)+(1-P)\left(x_{j} \otimes x_{k}\right) \gg_{\varepsilon}= \\
=\ll Y \otimes x_{i}, P\left(x_{j} \otimes x_{k}\right) \gg_{\varepsilon}+\ll Y \otimes x_{i},(1-P)\left(x_{j} \otimes x_{k}\right) \gg_{\varepsilon}
\end{gathered}
$$

Consider the second summand

$$
\begin{gathered}
\lambda_{i j}^{k}=\ll Y \otimes x_{i},(1-P)\left(x_{j} \otimes x_{k}\right) \gg_{\varepsilon}=\ll Y \otimes x_{i}, 1 \otimes x_{k} \gg_{\varepsilon}=\ll 1 \otimes Y, x_{k} \otimes x_{i} \gg_{W} \\
=\ll y, x_{k} \otimes x_{i} \gg_{\varepsilon}=\ll y, x_{k} \otimes x_{i} \gg
\end{gathered}
$$

So we can think of the numbers $\lambda_{i j}^{k}$ as being pre-determined. Let us focus on the first summand: we are going to find a suitable perturbation in order to determine those numbers. Let $\xi_{i}$ be the projection of the vector $Y \otimes x_{i}$ on $(V \otimes V) \otimes \mathbb{C}$ and $\eta_{i}=P \xi_{i}$. Then

$$
\ll Y \otimes x_{i}, P\left(x_{j} \otimes x_{k}\right) \gg_{\varepsilon}=\ll \xi_{i}, P\left(x_{j} \otimes x_{k}\right) \gg_{\varepsilon}=\ll \eta_{i}, x_{j} \otimes x_{k} \gg_{\varepsilon}
$$

So we would solve our problem if we found suitable $\eta_{i}$ 's. Now observe that they should verify the following

$$
\ll \eta_{i}, x_{j} \otimes x_{k} \gg_{\varepsilon}-\ll J_{\varepsilon} \eta_{j}, x_{k} \otimes x_{i} \gg_{\varepsilon}={\overline{\lambda_{j i}}}^{k}-\lambda_{i j}^{k}=: \theta_{i j}^{k}
$$


This is just a linear system. Before attempting to solve it, let us write it separately for the real and the imaginary part. We get

$$
\begin{gathered}
\operatorname{Re}\left(\theta_{i j}^{k}\right)=\operatorname{Re} \eta_{i}, x_{j} \otimes x_{k} \gg_{\varepsilon}-R e \ll J_{\varepsilon} \eta_{j}, x_{k} \otimes x_{i} \gg_{\varepsilon}= \\
=\frac{1}{2}\left(\ll \eta_{i}, x_{j} \otimes x_{k} \gg_{\varepsilon}+\overline{\ll \eta_{i}, x_{j} \otimes x_{k} \gg_{\varepsilon}}-\right. \\
\left.-\ll J_{\varepsilon} \eta_{j}, x_{k} \otimes x_{i} \gg_{\varepsilon}-\overline{\ll J_{\varepsilon} x_{j}, x_{k} \otimes x_{i} \gg_{\varepsilon}}\right)= \\
=\frac{1}{2}\left(\ll \eta_{i}, x_{j} \otimes x_{k} \gg_{\varepsilon}+\ll J_{\varepsilon} \eta_{i}, x_{k} \otimes x_{j} \gg_{\varepsilon}-\right. \\
\left.-\ll J_{\varepsilon} \eta_{j}, x_{k} \otimes x_{i} \gg_{\varepsilon}-\ll \eta_{j}, x_{i} \otimes x_{k} \gg_{\varepsilon}\right)= \\
=\ll \frac{\eta_{i}+J_{\varepsilon} \eta_{i}}{2}, \frac{x_{j} \otimes x_{k}+x_{k} \otimes x_{j}}{2} \gg_{\varepsilon}-\ll \frac{\eta_{j}+J_{\varepsilon} \eta_{j}}{2}, \frac{x_{k} \otimes x_{i}+x_{i} \otimes x_{k}}{2} \gg_{\varepsilon}+ \\
-\ll \frac{\eta_{i}-J_{\varepsilon} \eta_{i}}{2 i}, \frac{x_{j} \otimes x_{k}-x_{k} \otimes x_{j}}{2 i} \gg_{\varepsilon}-\ll \frac{\eta_{j}-J_{\varepsilon} \eta_{j}}{2 i}, \frac{x_{k} \otimes x_{i}-x_{i} \otimes x_{k}}{2 i} \gg_{\varepsilon}+ \\
=\ll \operatorname{Re}\left(\eta_{i}\right), \operatorname{Re}\left(x_{j} \otimes x_{k}\right) \gg_{\varepsilon}-\ll \operatorname{Im}\left(\eta_{i}\right), \operatorname{Im}\left(x_{j} \otimes x_{k}\right) \gg_{\varepsilon}+ \\
-\ll \operatorname{Re}\left(\eta_{j}\right), \operatorname{Re}\left(x_{k} \otimes x_{i}\right) \gg_{\varepsilon}-\ll \operatorname{Im}\left(\eta_{j}\right), \operatorname{Im}\left(x_{k} \otimes x_{i} \gg_{\varepsilon}\right.
\end{gathered}
$$

By an analogous calculation we get

$$
\begin{gathered}
\operatorname{Im}\left(\theta_{i j}^{k}\right)=\ll \operatorname{Im}\left(\eta_{i}\right), \operatorname{Re}\left(x_{j} \otimes x_{k}\right) \gg_{\varepsilon}+\ll \operatorname{Re}\left(\eta_{i}\right), \operatorname{Im}\left(x_{j} \otimes x_{k}\right) \gg_{\varepsilon}+ \\
+\ll \operatorname{Im}\left(\eta_{j}\right), \operatorname{Re}\left(x_{k} \otimes x_{i}\right) \gg_{\varepsilon}-\ll \operatorname{Re}\left(\eta_{j}\right), \operatorname{Im}\left(x_{k} \otimes x_{i} \gg_{\varepsilon}\right.
\end{gathered}
$$

Thus we have to solve the equations

$$
<\left(\ldots \operatorname{Re}\left(\eta_{i}\right), \operatorname{Im}\left(\eta_{i}\right), \ldots, \operatorname{Re}\left(\eta_{j}\right), \operatorname{Im}\left(\eta_{j}\right) \ldots\right), v_{i j}^{k}>=\operatorname{Re}\left(\theta_{i j}^{k}\right)
$$

and

$$
<\left(\ldots \operatorname{Re}\left(\eta_{i}\right), \operatorname{Im}\left(\eta_{i}\right), \ldots, \operatorname{Re}\left(\eta_{j}\right), \operatorname{Im}\left(\eta_{j}\right) \ldots\right), w_{i j}^{k}>=\operatorname{Im}\left(\theta_{i j}^{k}\right)
$$

being

$$
v_{i j}^{k}=\left(0, \ldots R e\left(x_{j} \otimes x_{k}\right),-\operatorname{Im}\left(x_{j} \otimes x_{k}\right), \ldots 0 \ldots,-\operatorname{Re}\left(x_{k} \otimes x_{i}\right),-\operatorname{Im}\left(x_{k} \otimes x_{i}\right), \ldots\right)
$$

and

$$
w_{i j}^{k}=\left(0, \ldots \operatorname{Im}\left(x_{j} \otimes x_{k}\right), \operatorname{Re}\left(x_{j} \otimes x_{k}\right), \ldots 0 \ldots-\operatorname{Im}\left(x_{k} \otimes x_{i}\right), \operatorname{Re}\left(x_{k} \otimes x_{i}\right) \ldots\right)
$$

where the non-zero components are exactly the ones corresponding to $i$ and $j$.

Now observe that $i, j$ are switchable everywhere and the case $i=j$ is trivial. So we 
can suppose $i<j$. Moreover, since the solvability of a linear system neither depend on permutations of the columns nor on multiplication by non-zero numbers, we can replace $v_{i j}^{k}$ and $w_{i j}^{k}$ by the following

$$
\begin{aligned}
& v_{i j}^{k}=\left(0, \ldots \operatorname{Re}\left(x_{j} \otimes x_{k}\right), \operatorname{Im}\left(x_{j} \otimes x_{k}\right), \ldots 0 \ldots-\operatorname{Re}\left(x_{k} \otimes x_{i}\right), \operatorname{Im}\left(x_{k} \otimes x_{i}\right), \ldots\right) \\
& w_{i j}^{k}=\left(0, \ldots-\operatorname{Im}\left(x_{j} \otimes x_{k}\right), \operatorname{Re}\left(x_{j} \otimes x_{k}\right), \ldots 0 \ldots \operatorname{Im}\left(x_{k} \otimes x_{i}\right), \operatorname{Re}\left(x_{k} \otimes x_{i}\right), \ldots\right)
\end{aligned}
$$

Such a re-writing concludes the first step.

\section{Step 2.}

The purpose of this step is to find a deformation of the $x_{i}$ 's (namely: a perturbation of the scalar product) such that the new $v_{i j}^{k}$ 's, $w_{i j}^{k}$ 's become linearly independent so that we can solve the equations in Step 1.

Suppose we have a linear combination which gives 0 :

$$
\sum_{2 \leq i<j \leq n, 2 \leq k \leq n} \alpha_{i j}^{k} v_{i j}^{k}+\sum_{2 \leq i<j \leq n, 2 \leq k \leq n} \beta_{i j}^{k} w_{i j}^{k}=0
$$

Now fix $i$ and look at this relation in the $i$-th component. We have of course the case $i<j$, but also a contribution that can be obtained from some $j^{\prime}<i$. So we can split the previous condition in the following ones:

$$
\begin{gathered}
\sum_{2 \leq i<j \leq n, 2 \leq k \leq n}\left(\alpha_{i j}^{k} \operatorname{Re}\left(x_{j} \otimes x_{k}\right)-\beta_{i j}^{k} \operatorname{Im}\left(x_{j} \otimes x_{k}\right)\right)+ \\
+\sum_{2 \leq j^{\prime}<i \leq n, 2 \leq k^{\prime} \leq n}\left(-\alpha_{j^{\prime} i}^{k^{\prime}} \operatorname{Re}\left(x_{k^{\prime}} \otimes x_{j^{\prime}}\right)+\beta_{j^{\prime} i}^{k^{\prime}} \operatorname{Im}\left(x_{k^{\prime}} \otimes x_{j^{\prime}}\right)\right)
\end{gathered}
$$

and

$$
\begin{gathered}
\sum_{2 \leq i<j \leq n, 2 \leq k \leq n}\left(\alpha_{i j}^{k} \operatorname{Im}\left(x_{j} \otimes x_{k}\right)+\beta_{i j}^{k} \operatorname{Re}\left(x_{j} \otimes x_{k}\right)\right)+ \\
+\sum_{2 \leq j^{\prime}<i \leq n, 2 \leq k^{\prime} \leq n}\left(\alpha_{j^{\prime} i}^{k^{\prime}} \operatorname{Im}\left(x_{k^{\prime}} \otimes x_{j^{\prime}}\right)+\beta_{j^{\prime} i}^{k^{\prime}} \operatorname{Re}\left(x_{k^{\prime}} \otimes x_{j^{\prime}}\right)\right)
\end{gathered}
$$

Now let $s_{i}$ semicircular (see [Vo]) and $\varepsilon^{\prime}>0$ small enough. Semicircularity guarantees that $\sqrt{1-\varepsilon^{\prime}} x_{i} \oplus \sqrt{\varepsilon^{\prime}} s_{i}=\tilde{x}_{i}$ are still an orthonormal basis, for any $\varepsilon^{\prime}>0$. The choice of $\varepsilon^{\prime}$ small enough guarantees that the scalar product

$$
\ll x_{i} \otimes x_{j}, x_{k} \otimes x_{l} \gg_{\varepsilon}:=\ll \tilde{x}_{i} \otimes \tilde{x}_{j}, \tilde{x}_{k} \otimes \tilde{x}_{l} \gg
$$


is an $\varepsilon$-deformation. Moreover observe that in this deformation $x_{i} \otimes x_{k}$ are linearly independent and independent from $x_{i} \otimes 1$. In particular $\operatorname{Re}\left(x_{i} \otimes x_{j}\right)$ and $\operatorname{Im}\left(x_{i} \otimes x_{j}\right)$ are linearly independent over the real numbers. It follows that we can separate real and imaginary part in the previous conditions and get

$$
\begin{aligned}
\sum_{2 \leq i<j \leq n, k=2, \ldots n} \alpha_{i j}^{k} \operatorname{Re}\left(x_{j} \otimes x_{k}\right)-\sum_{2 \leq j^{\prime}<i \leq n, k^{\prime}=2, \ldots n} \alpha_{j^{\prime} i}^{k^{\prime}} \operatorname{Re}\left(x_{k^{\prime}} \otimes x_{j^{\prime}}\right) & =0 \\
\sum_{2 \leq i<j \leq n, k=2, \ldots n} \alpha_{i j}^{k} \operatorname{Im}\left(x_{j} \otimes x_{k}\right)+\sum_{2 \leq j^{\prime}<i \leq n, k^{\prime}=2, \ldots n} \alpha_{j^{\prime} i}^{k^{\prime}} \operatorname{Im}\left(x_{k^{\prime}} \otimes x_{j^{\prime}}\right) & =0 \\
-\sum_{2 \leq i<j \leq n, k=2, \ldots n} \beta_{i j}^{k} \operatorname{Im}\left(x_{j} \otimes x_{k}\right)+\sum_{2 \leq j^{\prime}<i \leq n, k^{\prime}=2, \ldots n} \beta_{j^{\prime} i}^{k^{\prime}} \operatorname{Im}\left(x_{k^{\prime}} \otimes x_{j^{\prime}}\right) & =0 \\
\sum_{2 \leq i<j \leq n, k=2, \ldots n} \beta_{i j}^{k} \operatorname{Re}\left(x_{j} \otimes x_{k}\right)+\sum_{2 \leq j^{\prime}<i \leq n, k^{\prime}=2, \ldots n} \beta_{j^{\prime} i}^{k^{\prime}} \operatorname{Re}\left(x_{k^{\prime}} \otimes x_{j^{\prime}}\right) & =0
\end{aligned}
$$

Now, let us consider the first two conditions. If in the first sum $i<k$ or in the second sum $i>k^{\prime}$, the respective terms cannot cancel each other, so their coefficients must be zero. So one can have a term in the first sum equal to one in the second sum only in case $i>k, i<k^{\prime}, k$ corresponds to $j^{\prime}$ in the second sum and $k^{\prime}$ corresponds to $j$ in the first sum. In this case one has $\alpha_{i j}^{k}-\alpha_{j^{\prime} i}^{k^{\prime}}=0$ from the first condition and $\alpha_{i j}^{k}+\alpha_{j^{\prime} i}^{k^{\prime}}=0$ from the second one. It follows that these coefficients must be zero. Similarly we obtain that the $\beta$ 's are equal to zero.

\section{Step 3.}

Here we want to define the scalar product $\ll \cdot, \cdot \gg_{\varepsilon}$ whenever $Y$ appears twice. Recalling that the following properties have to be satisfied

$$
\begin{aligned}
& \text { 1. } \ll Y \otimes x_{i}, Y \otimes x_{j} \gg_{\varepsilon}=\overline{\ll x_{i} \otimes Y, x_{j} \otimes Y \gg_{\varepsilon}} \\
& \text { 2. } \ll Y \otimes x_{i}, Y \otimes x_{j} \gg_{\varepsilon}=\overline{\ll Y \otimes Y, x_{i} \otimes x_{j} \gg_{\varepsilon}}
\end{aligned}
$$

it follows that it will be enough to define the numbers $\ll Y \otimes x_{i}, Y \otimes x_{j} \gg_{\varepsilon}$ and $\ll Y \otimes x_{i}, x_{j} \otimes Y \gg_{\varepsilon}$. So, we can define the matrix $\left(\ll Y \otimes x_{i}, Y \otimes x_{j} \gg_{\varepsilon}\right)$ as any positive matrix (Indeed the perturbation in the second step causes $x_{i} \otimes x_{j}$ to be linearly independent with respect to $\ll \cdot, \cdot \gg_{\varepsilon}$ and then the second of the previous conditions gives no further constrictions). Finally we can set $\ll Y \otimes x_{i}, x_{j} \otimes Y \gg_{\varepsilon}=0$. 


\section{Forth Step:}

We can complete the proof very easily. Indeed we can set $\ll Y \otimes x_{i}, Y \otimes Y \gg_{\varepsilon}=0$, without contradictions. Finally, Bessel's inequality forces

$$
\ll Y \otimes Y, Y \otimes Y \gg_{\varepsilon} \geq \sum_{i, j}\left|\ll Y \otimes Y, x_{i} \otimes x_{j} \gg_{\varepsilon}\right|^{2}
$$

Also in this case there are no contradictions: it is enough to choose $\ll Y \otimes Y, Y \otimes Y \gg$ large enough. (What is the smallest possible value?)

Corollary 8. Let $V$ be a cyclic finite dimensional space with orthonormal basis $x_{1}, \ldots x_{n}$. Then for every $\varepsilon>0$ there exists a countably generated cyclic space $W_{\varepsilon}$, with cyclic structure $\ll \cdot, \cdot \gg_{\varepsilon}$, that verifies the following properties

1. $W$ extends $V$ as a vector space, i.e. the set $\left\{x_{1}, \ldots x_{n}\right\}$ extends to a basis $\left\{x_{1}, \ldots x_{n}, x_{n+1}, \ldots\right\}$ of $W$.

2. $\ll \cdot,\left.\cdot \gg_{\varepsilon}\right|_{V}$ is an $\varepsilon$-deformation of the cyclic structure on $V$.

3. $d_{\varepsilon}\left(x_{i} \otimes x_{j}, W_{\varepsilon} \otimes 1\right)=0$ for every $i, j \in \mathbb{N}$, where $d_{\varepsilon}(x, y)=\sqrt{\ll x-y, x-y \gg_{\varepsilon}}$

Proof. It is enough to iterate the previous lemma, taking $\varepsilon / 2^{n}$ at step $n$.

\section{Problems we were not able to solve}

Let $V$ be a separable cyclic vector space with orthonormal basis $\left\{x_{n}\right\}$. We can think about $x_{i}$ as the operator on $V$ defined by setting $x_{i}\left(x_{j}\right)=x_{i} \otimes x_{j}$. Prop 6 guarantees that this operator is well defined by linearity, but the problem is that it could be unbounded. Indeed $\left\|x_{i}\right\|^{2}=\sum_{n} \alpha_{i i}^{n}\left\|x_{n}\right\|^{2}$ could be infinite. So we have several open questions

1. Is the set of operators obtained in such a way a tracial algebra or at least as unbounded algebra of operators of type $I I$ in the sense of Inoue (see [In])?

2. Can we modify the proof of Prop $[$ in such a way that we get bounded operators?

3. What is the relation between this construction and that of Netzer and Thom (see [Ne-Th]), who seem to obtain similar objects? 


\section{Acknowledgement}

The authors are grateful to Robin Hillier for reading the draft of the paper and for suggesting a correction.

\section{References}

[Br] N.P. Brown, Connes' embedding problem and Lance's WEP, Int.Math.Rev.Notices (2004) 10, 501-510.

[Br2] , N.P.Brown, Topological Dynamical Systems Associated to $I I_{1}$-factors, preprint arXiv:1010.1214

[Ca-Pa] V. Capraro - L. Paunescu, Product between ultrafilter and applications to the Connes' embedding problem, preprint arXiv:math/0911.4978. Accepted by J. Oper. Theory.

[Co] A.Connes, Classification of injective factors. Cases $I I_{1}, I I_{\infty} . I I I_{1}$, Ann. of Math. (2) 104 (1976) no. 1, 73-115.

[Co-Dy] B. Collins and K. Dykema, Linearization of Connes' embedding problem, New York J. Math. 14 (2008) 617-641.

[El-Sz] G. Elek - E. Szabó, Hyperlinearity, essentially free actions and $L^{2}$-invariants. The sofic property, Math. Ann. 332 (2005) n.2, 421-441.

[Ha-Wi2] U. Haagerup and C. Winslow, The Effros-Marechal topology in the space of von Neumann algebras, II, J. Math. Anal. 171 (2000), 401-431.

[Ki] E. Kirchberg, On semisplit extensions, tensor products and exactness of group $C^{*}$ algebras, Inv. Math. 112 (1993), 449-489.

[In] A. Inoue, Tomita-Takesaki theory in algebras of unbounded operators, Lecture notes in Mathematics 1699, Springer Verlag.

[Ne-Th] T.Netzer and A. Thom, Tracial algebras and an embedding theorem, arxiv:math/1005.0823. 
[Oz] N. Ozawa, About the QWEP conjecture, Intern. J. Math. 15 (2004), 501-530.

[Pe] V. Pestov, Hyperlinear and sofic groups: a brief guide, The Bulletin of Symbolic Logic, vol. 14, number 4 (2008).

[Ra1] F. Rădulescu, The von Neumann algebras of the non-residually finite Baumslag group $<a, b \mid a b^{3} a^{-1}=b^{2}>$ embeds into $R^{\omega}$, Theta Ser. Adv. Math., vol. 9, Theta, Bucharest, 2008.

[Ra2] F. Rădulescu, A non-commutative, analytic version of Hilbert's 17-th problem in type $I I_{1}$ von Neumann algebras, To appear in Proceedings Theta Foundation.

[Ra3] F. Rădulescu, Convex sets associated with von Neumann algebras and Connes' approximate embedding problem, Math. Res. Lett. 6 (1999), no.2, 229-236.

[Vo] D.Voiculescu, Circular and semicircular system and free product factor, Progress in Math., vol.92, Birkhäuser, 1990.

[Vo2] D. Voiculescu, The analogues of entropy and of Fischer's information measure in free probability theory, I, Commun. Math. Phys. 155 (1993), 71-92.

[Vo3] D. Voiculescu, The analogues of entropy and of Fischer's information measure in free probability theory, II, Invent. Mat. 118 (1994), 411-440.

Valerio CAPRARO - University of Rome "Tor Vergata" - capraro@mat.uniroma2.it or valerio.capraro@virgilio.it

Florin RĂDULESCU - University of Rome "Tor Vergata" - radulesc@mat.uniroma2.it 